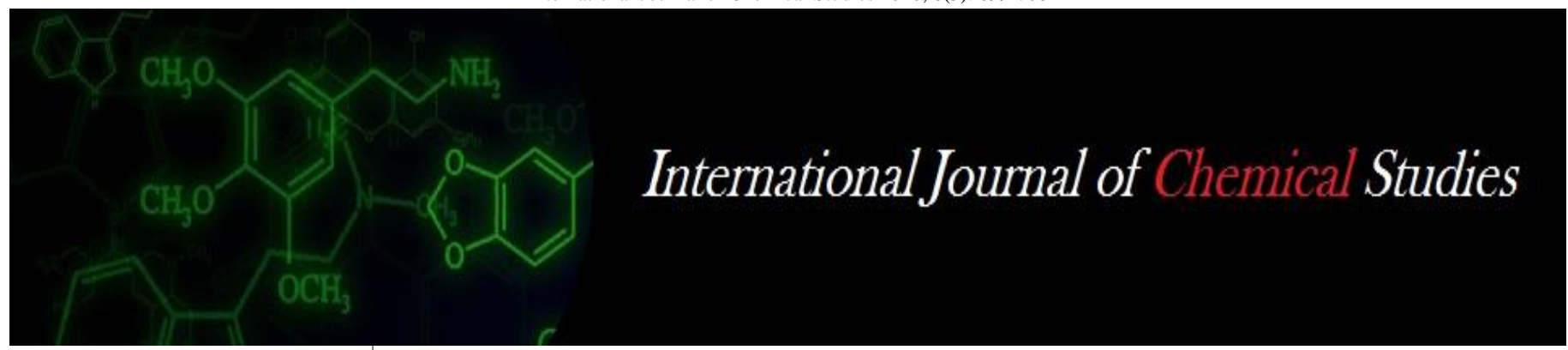

P-ISSN: 2349-8528

E-ISSN: 2321-4902

www.chemijournal.com

IJCS 2020; 8(5): 699-708

(C) 2020 IJCS

Received: 20-06-2020

Accepted: 18-08-2020

Siddharth Shankar Patre

Department of Agronomy

Indira Gandhi Krishi

Vishwavidyalaya, Raipur,

Chhattisgarh, India

GP Banjara

Department of Agronomy

Indira Gandhi Krishi

Vishwavidyalaya, Raipur,

Chhattisgarh, India

GK Shrivastava

Department of Agronomy

Indira Gandhi Krishi

Vishwavidyalaya, Raipur,

Chhattisgarh, India

\section{Ambika Tandon}

Department of Agronomy

Indira Gandhi Krishi

Vishwavidyalaya, Raipur,

Chhattisgarh, India

\section{SS Porte}

Department of Soil Science and

Agricultural Chemistry

Indira Gandhi Krishi

Vishwavidyalaya, Raipur,

Chhattisgarh, India
Corresponding Author: Siddharth Shankar Patre Department of Agronomy Indira Gandhi Krishi Vishwavidyalaya, Raipur, Chhattisgarh, India

\section{Effect of different herbicides on density and dry matter production of major weeds in chickpea (Cicer arietinum $\mathbf{L}$.)}

\author{
Siddharth Shankar Patre, GP Banjara, GK Shrivastava, Ambika Tandon \\ and SS Porte
}

DOI: https://doi.org/10.22271/chemi.2020.v8.i5j.10382

\begin{abstract}
A field experiment was conducted in the experimental plots of Instructional cum Research Farm, Indira Gandhi Krishi Vishwavidyalaya, Raipur, (C.G.) during rabi season 2019-20. The soil of experimental field was clayey (Vertisols) in texture, which was low, medium and high in available N (223 kg/ha.), P $(12.24 \mathrm{~kg} / \mathrm{ha}$.) and $\mathrm{K}(382.6 \mathrm{~kg} / \mathrm{ha}$.), respectively. The experiment was laid out in Randomized Block Design with four replications. The treatment of Topramezone 33.6 SC formulation (20.6 g a.i./ha) EPoE at $14 \mathrm{DAS}\left(\mathrm{T}_{1}\right)$, Topramezone $33.6 \mathrm{SC}$ formulation (20.6 g a.i./ha) EPoE at $21 \mathrm{DAS}\left(\mathrm{T}_{2}\right)$, Topramezone 33.6 SC formulation (25.7 g a.i./ha) EPoE at 14 DAS $\left(\mathrm{T}_{3}\right)$, Topramezone $33.6 \mathrm{SC}$ formulation $(25.7 \mathrm{~g}$ a.i./ha) EPoE at 21 DAS ( $\left.\mathrm{T}_{4}\right)$, Quizalofop-p-ethyl 5 EC (100 g a.i./ha) PoE at 25 DAS ( 55 ), Pendimethalin 30 EC formulation + Imazethapyr 2\% (Ready mix @ 1 kg/ha) PE + one Hoeing at 30 DAS (T6), Pendimethalin 30 EC formulation + Imazethapyr 2\% (Ready mix @ 1kg/ha) PE followed by Topramezone 33.6 SC (20.6 g a.i./ha) EPoE at 21 DAS ( $\left.\mathrm{T}_{7}\right)$, Pendimethalin $30 \mathrm{EC}$ formulation + Imazethapyr 2\% (Ready mix @ 1kg/ha) PE followed by Topramezone 33.6 SC (25.7 g a.i./ha) EPoE at 21 DAS ( $\left.\mathrm{T}_{8}\right)$,Weed free check (weeding at 20 and 40 DAS) $\left(\mathrm{T}_{9}\right)$, Weedy check $\left(\mathrm{T}_{10}\right)$. Results revealed that the experimental field Medicago denticulate, Chenopodium album, Echinoclhoa colona, and some other weeds were dominant and observed throughout the crop growth period. Weed free (weeding at 20 and 40 DAS) $\left(\mathrm{T}_{9}\right)$ followed by Pendimethalin $30 \mathrm{EC}+$ Imazethapyr $2 \%$ (Ready mix) @ $1.0 \mathrm{~kg} / \mathrm{ha} \mathrm{PE}+$ one hoeing at $30 \mathrm{DAS}\left(\mathrm{T}_{6}\right)$, Topramezone $33.6 \mathrm{SC}$ formulation $\left(20.6 \mathrm{~g}\right.$ i./ha) EPoE at $14 \mathrm{DAS}\left(\mathrm{T}_{1}\right)$, and Pendimethalin 30 EC formulation + Imazethapyr 2\% (Ready mix @ $1 \mathrm{~kg} / \mathrm{ha}$ ) PE followed by Topramezone 33.6 SC (20.6 g a.i./ha) EPoE at $21 \mathrm{DAS}\left(\mathrm{T}_{7}\right)$ were most appropriate for reducing weed density, weed dry matter production, lowest weed growth rate and gave highest weed control efficiency.
\end{abstract}

Keywords: Topramezone, chickpea (Cicer arietinum L.), weed control efficiency, weed density, weed dry matter production

\section{Introduction}

Chickpea (Cicer arietinum L.) is an important pulse crop grown and consumed all over the world, especially in the Afro-Asian countries. It is a good source of carbohydrates and protein, and protein quality is considered to be better than other pulses. Chickpea has significant amounts of all the essential amino acids except sulphur-containing amino acids, which can be complemented by adding cereals to the daily diet. Starch is the major storage carbohydrate followed by dietary fiber, oligosaccharides and simple sugars such as glucose and sucrose. Although lipids are present in low amounts, chickpea is rich in nutritionally important unsaturated fatty acids such as linoleic and oleic acids. $\beta$-sitosterol, campesterol and stigmasterol are important sterols present in chickpea oil. $\mathrm{Ca}, \mathrm{Mg}, \mathrm{P}$ and, especially, $\mathrm{K}$ are also present in chickpea seeds. Chickpea is a good source of important vitamins such as riboflavin, niacin, thiamin, folate and the vitamin A precursor $\beta$-carotene. Due to its nitrogen-fixing capacity, chickpea also plays a major role in increasing soil fertility.

In Indian cultivated area, grown over an area of 9.93 million ha with 9.53 million tons of production and an average productivity of $960 \mathrm{~kg}$ ha-1 (Anonymous, $2017 \mathrm{~A}$ ) ${ }^{[1]}$. The state of Chhattisgarh has better agro-ecological situation for the production of chickpea. It is grown in the state over an area of 349.81 thousand ha with an annual production of 556.31 thousand tons and an average output of $1116 \mathrm{~kg}$ ha-1 (Anonymous, 2017B) ${ }^{[2]}$. 
Chickpea is a weak competitor for weeds due to slow growth rates and limited leaf production at the early stage of crop growth and establishment if weed control is ignored under these conditions, resulting in yield losses of 40 to 87 per cent (Ratnam, 2013) ${ }^{[12]}$.

\section{Materials and Methods \\ 2.1 Study Site Description}

The field experiment was conducted at Instructional cum Research Farm, Indira Gandhi Krishi Vishwavidyalaya, Raipur, (C.G.) during rabi season 2019-20. Experimental site was situated at $21^{\circ} 4^{\prime} \mathrm{N}$ latitude and $81^{\circ} 35^{\prime} \mathrm{E}$ Longitude with an altitude of 290.20 meter above the mean sea level.

\subsection{Experimental Deteils}

The experiment was laid out in Randomized Block Design with four replications. The soil of experimental field was clayey (Vertisols) in texture, which was low, medium and high in available $\mathrm{N}$ (223 kg/ha.), $\mathrm{P}$ (12.24 kg/ha.) and $\mathrm{K}$ (382.6 kg/ha.), respectively. The treatment of Topramezone 33.6 SC formulation (20.6 g a.i./ha) EPoE at $14 \mathrm{DAS}\left(\mathrm{T}_{1}\right)$, Topramezone 33.6 SC formulation (20.6 g a.i./ha) EPoE at 21 DAS $\left(\mathrm{T}_{2}\right)$, Topramezone 33.6 SC formulation (25.7 $\mathrm{g}$ a.i./ha) $\mathrm{EPoE}$ at $14 \mathrm{DAS}\left(\mathrm{T}_{3}\right)$, Topramezone $33.6 \mathrm{SC}$ formulation (25.7 g a.i./ha) EPoE at $21 \mathrm{DAS}\left(\mathrm{T}_{4}\right)$, Quizalofop-p-ethyl 5 EC (100 g a.i./ha) PoE at 25 DAS $\left(\mathrm{T}_{5}\right)$, Pendimethalin $30 \mathrm{EC}$ formulation + Imazethapyr 2\% (Ready mix @ 1kg/ha) PE + one Hoeing at $30 \mathrm{DAS}\left(\mathrm{T}_{6}\right)$, Pendimethalin $30 \mathrm{EC}$ formulation + Imazethapyr 2\% (Ready mix @ 1kg/ha) PE followed by Topramezone 33.6 SC (20.6 g a.i./ha) EPoE at $21 \mathrm{DAS}\left(\mathrm{T}_{7}\right)$, Pendimethalin $30 \mathrm{EC}$ formulation + Imazethapyr 2\% (Ready mix @ 1kg/ha) PE followed by Topramezone 33.6 SC $(25.7 \mathrm{~g}$ a.i./ha) EPoE at $21 \mathrm{DAS}\left(\mathrm{T}_{8}\right)$, Weed free check (weeding at 20 and 40 DAS) $\left(\mathrm{T}_{9}\right)$, Weedy check $\left(\mathrm{T}_{10}\right)$. Herbicide dissolved thoroughly in water at the rate of 500 liter as ha- $\mathrm{ha}^{-1}$ as carrier and sprayed in their respective plots.

\subsection{Cultivation Ditails}

Chickpea (Cicer arietinum L.) variety Indira chana-1 was grown as test crop. It was released from IGKV, Raipur. It is a bold seeded variety and having brown seed colour. The crop duration is about 105-110 days Land preparation was performed when the soil reached working order. The field was prepared with plough drawn by tractor followed by harrowing to get a well-pulverized seed bed. Weed and crop residues have been stripped to get free seed bed for weed and stubble. It was eventually leveled with the help of a tractor-driven leveler. Recommended dose of nutrient 20:50:20 kg N : $\mathrm{P}_{2} \mathrm{O}_{5}$ : $\mathrm{K}_{2} \mathrm{O}$ ha $^{-1}$ was applied through diammonium phosphate (DAP), and muriate of potash (MOP), respectively as basal at the time of sowing in rows uniformly to each plot First irrigation was given by controlled flooding method just after sowing in order to ensure proper germination of the crop. Second irrigation was applied at 45 DAS.

The seed was sown mechanically in row on November 29, 2019. The spacing from row to row used a certified seed @ 80 $\mathrm{kg} \mathrm{ha}^{-1}$ was $30 \mathrm{~cm}$. The seeds were treated with carbendazim @ $2 \mathrm{~g} \mathrm{~kg}^{-1}$ of seed followed by seeds inoculated with Rhizobium culture @ $5 \mathrm{~g} \mathrm{~kg}^{-1}$ seeds before sowing to prevent the crop from soil and seed borne diseases. Seeds were manually sown after drying in shade. Weed management practices were adopted as per treatments. Two manual hand weeding were done at 20 and 40 days after sowing in hand weeding treatment and 30 DAS for $\mathrm{T}_{6}$. The harvesting was done manually with the help of sickle. The produce of each net plot was tied into bundle and allowed to sun drying in respective plots for two days. The harvested bundles were weighted with the help of spring balance and transported to threshing floor.

\subsection{Observation Recorded}

The important weed species associated with the chickpea crop in the experimental field were grouped in to two i.e. broadleaf and grassy.

\subsubsection{Weed density (Total and species wise)}

The weed density of different weed species was studied at 30 , 60, 90 DAS and at harvest. The weed study in each plot was made at random from five selected spots and for this purpose quadrate $\left(1 \mathrm{~m}^{-2}\right)$ was used. Only green weeds" samples were noted. Counting of weeds was done according to species and total population of weeds was also worked out. The data were recorded $\mathrm{m}^{-2}$ for statistical analysis.

\subsubsection{Dry matter production of weeds (Total and species wise)}

Dry matter production of weeds was recorded at 30, 60, 90 DAS and at harvest. The weeds of $1 \mathrm{~m}^{-2}$ quadrate were uprooted and roots were detached, sun dried and finally oven dried at $60{ }^{\circ} \mathrm{C}$ for 48 hours. The dry matter was recorded species wise and as total dry matter.

\subsubsection{Weed control efficiency (WCE)}

"The weed control efficiency was calculated at 30, 60, 90 DAS and at harvest on the basis of reduction in dry matter production of weeds in treated plots in comparison with weedy check and expressed in percentage as suggested by Mani et al. (1973) ${ }^{[10]}$.

$$
\mathrm{WCE}(\%)=\frac{\mathrm{DWC}-\mathrm{DWT}}{\mathrm{DWC}} \times 100
$$

Where,

$\mathrm{WCE}=$ Weed control efficiency $(\%)$

DWC $=$ Dry weight of weeds in weedy check plot $(\mathrm{g})$

DWT $=$ Dry weight of weeds in treated plot $(\mathrm{g})$

\subsubsection{Transformation of data}

"Data on weed count and weed dry weight showed high variation. To make the analysis of variance more valid the data on weed count and weed dry weight was subjected to square root transformation by using formula $\sqrt{\mathrm{x}}+0.5$ (Chandel, 1984) ${ }^{[6]}$.

\section{Results and Discussion}

\subsection{Total and species wise weed density $\left(\mathrm{No.m}^{-2}\right)$}

Its total weed density of Medicago denticulata, Echinochloa colona, Chenopodium album and many others were recorded at 30, 60, 90 DAS and mentioned in Table: 1. at harvest and. At all the observing stages the overall weed density was greatly affected by various weed control treatments.

"At 30 DAS, lowest total weed density was observed under the treatment of weed free (hand weeding at 20 and 40 DAS) (T9), however, it was at par to the treatment of Pendimethalin $30 \mathrm{EC}+$ Imazethapyr 2\% (Ready mix) @ $1.0 \mathrm{~kg} \mathrm{ha}^{-1} \mathrm{PE}+$ one hoeing at $30 \mathrm{DAS}\left(\mathrm{T}_{6}\right)$, Topramezone 33.6 $\mathrm{SC}$ formulation (20.6 g a.i./ha) EPoE at 14 DAS ( $\left.\mathrm{T}_{1}\right)$, Pendimethalin $30 \mathrm{EC}$ formulation + Imazethapyr 2\% (Ready mix @ $1 \mathrm{~kg} / \mathrm{ha}$ ) PE $f b$ Topramezone 33.6 SC (20.6 g a.i./ha) EPoE at $21 \mathrm{DAS}\left(\mathrm{T}_{7}\right)$, 
and Topramezone 33.6 SC formulation(25.7 g a.i./ha) EPoE at 14 DAS $\left(\mathrm{T}_{3}\right)$, Comparably, at different observation intervals, i.e. at 60, 90 DAS and at harvest, relatively lower total weed density was reported under hand weeding at 20 and 40 DAS (T9), which was considerably greater than other treatments, while under" weed control (T10) it was larger than the rest of the treatments.

"Among the herbicidal treatments, significantly lower weed density recorded under the treatment of Pendimethalin $30 \mathrm{EC}$ + Imazethapyr 2\% (Ready mix) @ $1.0 \mathrm{~kg} \mathrm{ha}^{-1} \mathrm{PE}+$ one hoeing at 30 DAS $\left(\mathrm{T}_{6}\right)$, which was at par with Topramezone $33.6 \mathrm{SC}$ formulation (20.6 $\mathrm{g}$ a.i./ha) $\mathrm{EPoE}$ at $14 \mathrm{DAS}\left(\mathrm{T}_{1}\right)$, and Pendimethalin 30 EC formulation + Imazethapyr 2\% (Ready mix @ 1kg/ha) PE fb Topramezone 33.6 SC (20.6 g a.i./ha)
EPoE at 21 DAS $\left(\mathrm{T}_{7}\right)$ and Topramezone 33.6 SC formulation (25.7 g a.i./ha) EPoE at $14 \mathrm{DAS}\left(\mathrm{T}_{3}\right)$, respectively, and significantly superior rest of the treatments. Then it can be reported on the base of the above results that the weed density trend was in ascending order from 30 DAS to harvest point. The use of herbicides and weeding by hand reduced significantly the density of weeds at the early stage of crop yields. In fact, weed infestation increased significantly under control plot (T10) up to harvest, and highest weed density was observed during the crop growth cycle in control plot (T10), as no control measure was adopted. Such results are consistent with the" Butter and Aggarwal (2008) ${ }^{[4]}$, Kumar (2010) ${ }^{[9]}$ and Singh et al. (2014) ${ }^{[15]}$ find

Table 1: Total weed population in chickpea as influenced by different weed control measures

\begin{tabular}{|c|c|c|c|c|}
\hline \multirow[t]{2}{*}{ Treatment } & \multicolumn{4}{|c|}{ Weed population $\left(\right.$ No.m $\left.^{-2}\right)$} \\
\hline & \multicolumn{4}{|c|}{30 DAS 60 DAS 90 DAS At harvest } \\
\hline \multirow[t]{2}{*}{$\mathrm{T}_{1}$ Topramezone $33.6 \mathrm{SC}$ formulation(20.6 g a.i./ha) EPoE at $14 \mathrm{DAS}$} & 5.70 & 5.87 & 5.43 & 5.26 \\
\hline & $(32.10)$ & $(34.05)$ & $(29.10)$ & $(27.20)$ \\
\hline \multirow[t]{2}{*}{$\mathrm{T}_{2}$ Topramezone $33.6 \mathrm{SC}$ formulation(20.6 $\mathrm{g}$ a.i./ha) EPoE at $21 \mathrm{DAS}$} & 6.17 & 6.37 & 6.08 & 5.82 \\
\hline & $(37.55)$ & $(40.05)$ & $(36.55)$ & $(33.45)$ \\
\hline \multirow[t]{2}{*}{$\mathrm{T}_{3}$ Topramezone 33.6 SC formulation(25.7 g a.i./ha) EPoE at $14 \mathrm{DAS}$} & 6.09 & 6.13 & 5.70 & 5.47 \\
\hline & $(36.60)$ & $(37.20)$ & $(32.15)$ & $(29.55)$ \\
\hline \multirow{2}{*}{$\mathrm{T}_{4}$ Topramezone $33.6 \mathrm{SC}$ formulation (25.7 g a.i./ha) EPoE at $21 \mathrm{DAS}$} & 6.22 & 6.40 & 6.24 & 5.92 \\
\hline & $(38.25)$ & $(40.55)$ & $(38.45)$ & $(34.60)$ \\
\hline \multirow{2}{*}{ T5 Quizalofop-p-ethyl 5 EC (100 g a.i./ha) PoE at 25 DAS } & 6.29 & 6.48 & 6.37 & 5.99 \\
\hline & $(39.03)$ & $(40.80)$ & $(40.20)$ & $(35.50)$ \\
\hline \multirow{2}{*}{$\begin{array}{c}\mathrm{T}_{6} \text { Pendimethalin } 30 \mathrm{EC} \text { formulation + Imazethapyr } 2 \% \text { (Ready mix @ } 1 \mathrm{~kg} / \mathrm{ha} \text { ) PE + one Hoeing /hand } \\
\text { weeding at } 30 \text { DAS }\end{array}$} & 4.93 & 4.98 & 4.00 & 3.79 \\
\hline & $(23.85)$ & $(23.50)$ & $(15.60)$ & $(13.85)$ \\
\hline \multirow{2}{*}{$\begin{array}{c}\left.\text { T }_{7} \text { Pendimethalin } 30 \text { EC formulation + Imazethapyr 2\% (Ready mix @ } 1 \mathrm{~kg} / \mathrm{ha}\right) \text { PE followed by } \\
\text { Topramezone 33.6 SC (20.6 g a.i./ha) EPoE at } 21 \text { DAS }\end{array}$} & 6.01 & 6.07 & 5.67 & 5.06 \\
\hline & $(35.73)$ & $(42.20)$ & $(31.75)$ & $(25.55)$ \\
\hline \multirow{2}{*}{$\begin{array}{c}\text { T8 Pendimethalin } 30 \text { EC formulation + Imazethapyr 2\% (Ready mix @ 1kg/ha) PE followed by } \\
\text { Topramezone 33.6 SC (25.7 g a.i./ha) EPoE at } 21 \text { DAS }\end{array}$} & 6.13 & 6.26 & 5.73 & 5.58 \\
\hline & $(37.05)$ & $(39.75)$ & $(32.50)$ & $(30.70)$ \\
\hline \multirow{2}{*}{$\mathrm{T}_{9}$ Weed free check (hand weeding at 20 and $40 \mathrm{DAS}$ ) } & 4.81 & 4.71 & 3.87 & 3.62 \\
\hline & $(22.70)$ & $(21.85)$ & $(14.63)$ & $(12.85)$ \\
\hline \multirow{2}{*}{$\mathrm{T}_{10}$ Weedy check } & 9.45 & 10.25 & 9.32 & 9.14 \\
\hline & $(88.90)$ & (104.6) & $(86.41)$ & $(83.15)$ \\
\hline $\mathrm{SEm} \pm$ & 0.11 & 0.18 & 0.17 & 0.19 \\
\hline $\mathrm{CD}(\mathrm{P}=0.05)$ & 0.34 & 0.53 & 0.50 & 0.55 \\
\hline
\end{tabular}

\subsection{Species wise weed density (No. $\left.\mathrm{m}^{-2}\right)$}

\subsubsection{Medicago denticulata $\left(\mathrm{No}^{-\mathrm{m}^{-2}}\right)$}

"The slightly lowest density of Medicago denticulata in weedfree treatment was reported at 30 DAS (hand weeding at 20 and 40 DAS) (T9), however, it was at par to the treatment of Pendimethalin 30 EC + Imazethapyr 2\% (Ready mix) @ 1.0 $\mathrm{kg} \mathrm{ha}^{-1} \mathrm{PE}+$ one hoeing at $30 \mathrm{DAS}\left(\mathrm{T}_{6}\right)$, Topramezone 33.6 $\mathrm{SC}$ formulation (20.6 $\mathrm{g}$ a.i./ha) EPoE at $14 \mathrm{DAS}\left(\mathrm{T}_{1}\right)$, Pendimethalin 30 EC formulation + Imazethapyr 2\% (Ready mix@1kg/ha) PE" fb Topramezone 33.6 SC (20.6 g a.i./ha) EPoE at 21 DAS $\left(\mathrm{T}_{7}\right)$, and Topramezone 33.6 SC formulation (25.7 g a.i./ha) EPoE at 14 DAS ( $\left.\mathrm{T}_{3}\right)$ (Table 2. depicted).

"At 60, 90 DAS and at harvest, significantly lowest density was noted under weed free (hand weeding at 20 and 40 DAS
$\left(\mathrm{T}_{9}\right)$ which was significantly superior over other treatments. Among the herbicidal treatments, significantly lower density was observed in Pendimethalin $30 \mathrm{EC}+$ Imazethapyr 2\% (Ready mix) @ $1.0 \mathrm{~kg} \mathrm{ha}^{-1} \mathrm{PE}+$ one hoeing at $30 \mathrm{DAS}$ ( $\mathrm{T}_{6}$ ), Topramezone 33.6 SC formulation (20.6 g a.i./ha) EPoE at 14 DAS $\left(\mathrm{T}_{1}\right)$, Pendimethalin 30 EC formulation + Imazethapyr 2\% (Ready mix @ 1kg/ha) PE fb Topramezone 33.6 SC (20.6 g a.i./ha) EPoE at 21 DAS $\left(\mathrm{T}_{7}\right)$, and Topramezone 33.6 SC formulation(25.7 g a.i./ha) EPoE at 14 DAS $\left(\mathrm{T}_{3}\right)$ Under weedy check (T10) the maximum density of Medicago denticulata (T10) was reported.Similar results was reported" by Singh et al. (2014) ${ }^{[15]}$. 


\begin{tabular}{|c|c|c|c|c|}
\hline Treatment & \multirow{2}{*}{\multicolumn{4}{|c|}{\begin{tabular}{|c|} 
Weed population $\left(\right.$ No.m $\left.^{-2}\right)$ \\
30 DAS60 DAS 90 DAS At harvest
\end{tabular}}} \\
\hline & & & & \\
\hline \multirow{2}{*}{$\mathrm{T}_{1}$ Topramezone $33.6 \mathrm{SC}$ formulation (20.6 g a.i./ha) PoE at $14 \mathrm{DAS}$} & 4.06 & 4.18 & 3.88 & 3.76 \\
\hline & \begin{tabular}{|l|}
$(16.05)$ \\
\end{tabular} & (17.00) & (14.60) & (13.65) \\
\hline \multirow{2}{*}{$\mathrm{T}_{2}$ Topramezone $33.6 \mathrm{SC}$ formulation (20.6 g a.i./ha) PoE at $21 \mathrm{DAS}$} & 4.37 & 4.47 & 4.33 & 4.14 \\
\hline & $(18.65)$ & $(19.50)$ & $(18.30)$ & $(16.70)$ \\
\hline \multirow{2}{*}{$\mathrm{T}_{3}$ Topramezone $33.6 \mathrm{SC}$ formulation (25.7 g a.i./ha) PoE at $14 \mathrm{DAS}$} & 4.33 & 4.34 & 4.06 & 3.89 \\
\hline & $(18.30)$ & $(18.45)$ & $(16.10)$ & $(14.70)$ \\
\hline \multirow{2}{*}{$\mathrm{T}_{4}$ Topramezone $33.6 \mathrm{SC}$ formulation ( $25.7 \mathrm{~g}$ a.i./ha) EPoE at $21 \mathrm{DAS}$} & 4.43 & 4.50 & 4.45 & 4.22 \\
\hline & $(19.15)$ & $(19.85)$ & $(19.35)$ & $(17.30)$ \\
\hline \multirow{2}{*}{$\mathrm{T}_{5}$ Quizalofop-p-ethyl $5 \mathrm{EC}$ (100 g a.i./ha) PoE at $25 \mathrm{DAS}$} & 4.47 & 4.57 & 4.53 & 4.27 \\
\hline & $(19.53)$ & $(20.40)$ & $(20.10)$ & $(17.75)$ \\
\hline \multirow{2}{*}{$\begin{array}{c}\text { T6 Pendimethalin } 30 \text { EC formulation + Imazethapyr 2\% (Ready mix @ 1kg/ha) PE + one Hoeing /hand } \\
\text { weeding at } 30 \text { DAS }\end{array}$} & 3.59 & 3.50 & 2.90 & 2.74 \\
\hline & $(12.40)$ & $(11.75)$ & (7.95) & $(7.00)$ \\
\hline \multirow{2}{*}{$\begin{array}{c}\mathrm{T}_{7} \text { Pendimethalin } 30 \text { EC formulation + Imazethapyr 2\% (Ready mix @ 1kg/ha) PE followed by } \\
\text { Topramezone 33.6 SC (20.6 g a.i./ha) EPoE at } 21 \text { DAS }\end{array}$} & 4.32 & 4.32 & 4.05 & 3.21 \\
\hline & $(18.18)$ & $(18.35)$ & $(15.90)$ & $(10.90)$ \\
\hline \multirow{2}{*}{$\begin{array}{c}\text { T8 Pendimethalin } 30 \text { EC formulation + Imazethapyr 2\% (Ready mix @ } 1 \mathrm{~kg} / \mathrm{ha} \text { ) PE followed by } \\
\text { Topramezone 33.6 SC (25.7 g a.i./ha) EPoE at } 21 \text { DAS }\end{array}$} & 4.34 & 4.40 & 4.08 & 3.97 \\
\hline & $(18.35)$ & $(18.90)$ & $(16.25)$ & $(15.30)$ \\
\hline \multirow{2}{*}{$\mathrm{T}_{9}$ Weed free check (hand weeding at 20 and 40 DAS ) } & 3.45 & 3.37 & 2.78 & 2.61 \\
\hline & $(11.40)$ & $(10.85)$ & $(7.33)$ & $(6.45)$ \\
\hline \multirow{2}{*}{$\mathrm{T}_{10}$ Weedy check } & 6.64 & 7.26 & 6.61 & 6.48 \\
\hline & $(43.65)$ & $(52.30)$ & (43.20) & $(41.55)$ \\
\hline SEm \pm & 0.09 & 0.14 & 0.12 & 0.22 \\
\hline $\mathrm{CD}(\mathrm{P}=0.05)$ & 0.27 & 0.41 & 0.36 & 0.63 \\
\hline
\end{tabular}

\subsubsection{Echinochloa colona $\left(\right.$ No. $\left.\mathrm{m}^{-2}\right)$}

At 30 DAS, the density of Echinochloa colona was minimum under the treatment of weed free (hand weeding at 20 and 40 DAS) ( $\mathrm{T}_{9}$ ), however, it was at par to the treatment of Pendimethalin 30 EC + Imazethapyr 2\% (Ready mix) @ 1.0 $\mathrm{kg} \mathrm{ha}^{-1} \mathrm{PE}+$ one hoeing at $30 \mathrm{DAS}\left(\mathrm{T}_{6}\right)$, Topramezone 33.6 $\mathrm{SC}$ formulation (20.6 g a.i./ha) EPoE at $14 \mathrm{DAS}\left(\mathrm{T}_{1}\right)$, Pendimethalin $30 \mathrm{EC}$ formulation + Imazethapyr 2\% (Ready mix @ 1kg/ha) PE fb Topramezone 33.6 SC (20.6 g a.i./ha) EPoE at 21 DAS $\left(\mathrm{T}_{7}\right)$, and Topramezone $33.6 \mathrm{SC}$ formulation(25.7 g a.i./ha) EPoE at 14 DAS $\left(\mathrm{T}_{3}\right)$ (Table 3).

"At 60, 90 DAS and at harvest, the weed free (hand weeding at 20 and 40 DAS) (T9) recorded significantly lowest
Echinochloa colona density. Under weedy check (T10) the maximum density was recorded. Among the herbicidal treatments, at 60, 90 DAS and at harvest, treatment of Pendimethalin 30 EC + Imazethapyr 2\% (Ready mix) @1.0 $\mathrm{kg} \mathrm{ha}^{-1} \mathrm{PE}+$ one hoeing at 30 DAS $\left(\mathrm{T}_{6}\right)$ Topramezone 33.6 $\mathrm{SC}$ formulation (20.6 $\mathrm{g}$ a.i./ha) EPoE at $14 \mathrm{DAS}\left(\mathrm{T}_{1}\right)$, Pendimethalin 30 EC formulation + Imazethapyr 2\% (Ready mix @ 1kg/ha) PE $f b$ Topramezone 33.6 SC (20.6 g a.i./ha) EPoE at 21 DAS $\left(\mathrm{T}_{7}\right)$, and Topramezone $33.6 \mathrm{SC}$ formulation(25.7 $\mathrm{g}$ a.i./ha) $\mathrm{EPoE}$ at $14 \mathrm{DAS}\left(\mathrm{T}_{3}\right)$ were at par to the weed free (hand weeding at 20 and $40 \mathrm{DAS})\left(\mathrm{T}_{9}\right)$.

Table 3: Density of Echinochloa colona in chickpea as influenced by different weed control measures

\begin{tabular}{|c|c|c|c|c|}
\hline \multirow{2}{*}{ Treatment } & \multirow{2}{*}{\multicolumn{4}{|c|}{\begin{tabular}{|c|} 
Weed population $\left(\right.$ No.m $\left.^{-2}\right)$ \\
30 DAS 60 DAS 90 DAS At harvest
\end{tabular}}} \\
\hline & & & & \\
\hline \multirow{2}{*}{$\mathrm{T}_{1}$ Topramezone $33.6 \mathrm{SC}$ formulation(20.6 $\mathrm{g}$ a.i./ha) EPoE at $14 \mathrm{DAS}$} & 2.92 & 3.00 & 2.79 & 2.69 \\
\hline & \begin{tabular}{|l}
$(8.05)$ \\
\end{tabular} & \begin{tabular}{|l|l}
$(8.50)$ \\
\end{tabular} & \begin{tabular}{|l|l}
$(7.30)$ \\
\end{tabular} & $(6.75)$ \\
\hline \multirow{2}{*}{$\mathrm{T}_{2}$ Topramezone $33.6 \mathrm{SC}$ formulation(20.6 $\mathrm{g}$ a.i./ha) EPoE at $21 \mathrm{DAS}$} & 3.12 & 3.29 & 3.10 & 2.98 \\
\hline & \begin{tabular}{|l}
$(9.25)$ \\
\end{tabular} & $(10.35)$ & \begin{tabular}{|l|l|}
$(9.15)$ \\
\end{tabular} & $(8.40)$ \\
\hline \multirow{2}{*}{$\mathrm{T}_{3}$ Topramezone $33.6 \mathrm{SC}$ formulation( $25.7 \mathrm{~g}$ a.i./ha) EPoE at $14 \mathrm{DAS}$} & 3.10 & 3.15 & 2.92 & 2.81 \\
\hline & $(9.15)$ & $(9.45)$ & $(8.05)$ & $(7.40)$ \\
\hline \multirow{2}{*}{$\mathrm{T}_{4}$ Topramezone $33.6 \mathrm{SC}$ formulation (25.7 $\mathrm{g}$ a.i./ha) EPoE at $21 \mathrm{DAS}$} & 3.15 & 3.30 & 3.18 & 3.04 \\
\hline & $(9.45)$ & $(10.40)$ & $(9.65)$ & $(8.75)$ \\
\hline \multirow{2}{*}{$\mathrm{T}_{5}$ Quizalofop-p-ethyl 5 EC (100 g a.i./ha) PoE at 25 DAS } & 3.17 & 3.33 & 3.24 & 3.06 \\
\hline & \begin{tabular}{|l|}
$(9.55)$ \\
\end{tabular} & $(10.60)$ & $(10.05)$ & $(8.90)$ \\
\hline \multirow{2}{*}{$\begin{array}{c}\text { T6 Pendimethalin } 30 \text { EC formulation + Imazethapyr 2\% (Ready mix @ 1kg/ha) PE + one Hoeing /hand } \\
\text { weeding at } 30 \text { DAS }\end{array}$} & 2.47 & 2.67 & 2.10 & 1.99 \\
\hline & $(5.60)$ & $(6.65)$ & (3.95) & $(3.45)$ \\
\hline \multirow{2}{*}{$\begin{array}{c}\mathrm{T}_{7} \text { Pendimethalin } 30 \mathrm{EC} \text { formulation + Imazethapyr 2\% (Ready mix @ 1kg/ha) PE followed by } \\
\text { Topramezone 33.6 SC (20.6 g a.i./ha) EPoE at } 21 \text { DAS }\end{array}$} & 3.06 & 3.09 & 2.91 & 2.80 \\
\hline & $(8.90)$ & $(9.15)$ & \begin{tabular}{|l|}
$(8.00)$ \\
\end{tabular} & $(7.35)$ \\
\hline \multirow{2}{*}{$\begin{array}{c}\left.\mathrm{T}_{8} \text { Pendimethalin } 30 \mathrm{EC} \text { formulation + Imazethapyr 2\% (Ready mix @ } 1 \mathrm{~kg} / \mathrm{ha}\right) \text { PE followed by } \\
\text { Topramezone 33.6 SC (25.7 g a.i./ha) EPoE at } 21 \text { DAS }\end{array}$} & 3.11 & 3.24 & 2.94 & 2.86 \\
\hline & \begin{tabular}{|l|}
$(9.20)$ \\
\end{tabular} & $(10.00)$ & $(8.15)$ & $(7.70)$ \\
\hline \multirow{2}{*}{$\mathrm{T}_{9}$ Weed free check (hand weeding at 20 and 40 DAS ) } & 2.49 & 2.44 & 2.03 & 1.91 \\
\hline & $(5.70)$ & $(5.45)$ & (3.65) & $(3.20)$ \\
\hline \multirow{2}{*}{$\mathrm{T}_{10}$ Weedy check } & 4.80 & 5.16 & 4.70 & 4.61 \\
\hline & $(22.60)$ & $(26.20)$ & (21.61) & (20.80) \\
\hline SEm \pm & 0.07 & 0.09 & 0.08 & 0.07 \\
\hline $\mathrm{CD}(\mathrm{P}=0.05)$ & 0.20 & 0.27 & 0.25 & 0.23 \\
\hline
\end{tabular}

3.2.3 Chenopodium album (No. $\mathrm{m}^{-2}$ )

At 30 DAS, the minimum density of Chenopodium album was observed in the treatment of weed free (hand weeding at 20 and 40 DAS) ( $\left.T_{9}\right)$, however, it was at par to the treatment of Pendimethalin 30 EC + Imazethapyr 2\% (Ready mix) @ 1.0 $\mathrm{kg} \mathrm{ha}^{-1} \mathrm{PE}+$ one hoeing at $30 \mathrm{DAS}\left(\mathrm{T}_{6}\right)$, Topramezone 33.6 
SC formulation (20.6 g a.i./ha) EPoE at $14 \mathrm{DAS}\left(\mathrm{T}_{1}\right)$, Pendimethalin 30 EC formulation + Imazethapyr 2\% (Ready mix @ 1kg/ha) PE"fb Topramezone 33.6 SC (20.6 g a.i./ha) $\mathrm{EPoE}$ at $21 \mathrm{DAS}\left(\mathrm{T}_{7}\right)$, and Topramezone 33.6 SC formulation (25.7 g a.i./ha) EPoE at 14 DAS ( $\left.\mathrm{T}_{3}\right)$ (Table 4).

Significantly lowest density "was recorded under 60, 90 DAS and at harvest weed free (hand weeding at 20 and 40 DAS) $\left(\mathrm{T}_{9}\right)$. The maximum Chenopodium album density was recorded under weedy check $\left(\mathrm{T}_{10}\right)$. Among herbicidal treatments, at $60 \mathrm{DAS}, 90 \mathrm{DAS}$ and at harvest, treatment of Pendimethalin $30 \mathrm{EC}+$ Imazethapyr 2\% (Ready mix) @ 1.0 $\mathrm{kg} \mathrm{ha}^{-1} \mathrm{PE}+$ one hoeing at $30 \mathrm{DAS}\left(\mathrm{T}_{6}\right)$, Topramezone 33.6 $\mathrm{SC}$ formulation (20.6 g a.i./ha) EPoE at $14 \mathrm{DAS}\left(\mathrm{T}_{1}\right)$, Pendimethalin $30 \mathrm{EC}$ formulation + Imazethapyr 2\% (Ready mix @ 1kg/ha) PE $f b$ Topramezone 33.6 SC (20.6 g a.i./ha) $\mathrm{EPoE}$ at 21 DAS $\left(\mathrm{T}_{7}\right)$, and Topramezone 33.6 $\mathrm{SC}$ formulation (25.7 $\mathrm{g}$ a.i./ha) EPoE at $14 \mathrm{DAS}\left(\mathrm{T}_{3}\right)$ were at par to the weed free (hand weeding at 20 and $40 \mathrm{DAS})$ " ( $\left.\mathrm{T}_{9}\right)$, in descending order. However, density of Chenopodium album under all the herbicidal treatments was significantly lower than control plot and other treatments.

Table 4: Density of Chenopodium album in chickpea as influenced by different weed control measures

\begin{tabular}{|c|c|c|c|c|}
\hline \multirow[t]{2}{*}{ Treatment } & \multicolumn{4}{|c|}{ Weed population (No./m²) } \\
\hline & 30 DAS & 60 DAS & 90 DAS & t harvest \\
\hline \multirow{2}{*}{$\mathrm{T}_{1}$ Topramezone $33.6 \mathrm{SC}$ formulation(20.6 g a.i./ha) EPoE at $14 \mathrm{DAS}$} & 2.30 & 2.37 & 2.20 & 2.13 \\
\hline & $(4.80)$ & $(5.15)$ & $(4.35)$ & $(4.05)$ \\
\hline \multirow{2}{*}{$\mathrm{T}_{2}$ Topramezone $33.6 \mathrm{SC}$ formulation(20.6 g a.i./ha) EPoE at $21 \mathrm{DAS}$} & 2.51 & 2.58 & 2.45 & 2.34 \\
\hline & $(5.80)$ & $(6.15)$ & $(5.50)$ & $(5.00)$ \\
\hline \multirow{2}{*}{$\mathrm{T}_{3}$ Topramezone 33.6 SC formulation(25.7 g a.i./ha) EPoE at $14 \mathrm{DAS}$} & 2.45 & 2.45 & 2.30 & 2.22 \\
\hline & $(5.50)$ & \begin{tabular}{|l|l|}
$(5.55)$ \\
\end{tabular} & \begin{tabular}{|l|l}
$(4.80)$ \\
\end{tabular} & $(4.45)$ \\
\hline \multirow{2}{*}{$\mathrm{T}_{4}$ Topramezone formulation $33.6 \mathrm{SC}$ (25.7 $\mathrm{g}$ a.i./ha) EPoE at $21 \mathrm{DAS}$} & 2.52 & 2.59 & 2.51 & 2.38 \\
\hline & $(5.85)$ & $(6.20)$ & $(5.80)$ & $(5.15)$ \\
\hline \multirow{2}{*}{$\mathrm{T}_{5}$ Quizalofop-p-ethyl $5 \mathrm{EC}$ (100 g a.i./ha) PoE at $25 \mathrm{DAS}$} & 2.55 & 2.61 & 2.55 & 2.41 \\
\hline & $(6.00)$ & $(6.35)$ & $(6.00)$ & $(5.30)$ \\
\hline \multirow{2}{*}{$\begin{array}{c}\text { T6 Pendimethalin } 30 \text { EC formulation + Imazethapyr 2\% (Ready mix @ 1 kg/ha) PE + one Hoeing /hand } \\
\text { weeding at } 30 \text { DAS }\end{array}$} & 1.99 & 2.02 & 1.63 & 1.60 \\
\hline & $(3.47)$ & $(3.60)$ & $(2.15)$ & $(2.05)$ \\
\hline \multirow{2}{*}{$\begin{array}{c}\mathrm{T}_{7} \text { Pendimethalin } 30 \text { EC formulation + Imazethapyr 2\% (Ready mix @ } 1 \mathrm{~kg} / \mathrm{ha} \text { ) PE followed by } \\
\text { Topramezone 33.6 SC (20.6 g a.i./ha) EPoE at } 21 \text { DAS }\end{array}$} & 2.43 & 2.46 & 2.29 & 2.20 \\
\hline & $(5.40)$ & $(5.60)$ & $(4.75)$ & $(4.35)$ \\
\hline \multirow{2}{*}{$\begin{array}{c}\text { T8 Pendimethalin } 30 \text { EC formulation + Imazethapyr 2\% (Ready mix @ } 1 \mathrm{~kg} / \mathrm{ha} \text { ) PE followed by } \\
\text { Topramezone 33.6 SC (25.7 g a.i./ha) EPoE at } 21 \mathrm{DAS}\end{array}$} & 2.50 & 2.54 & 2.31 & 2.25 \\
\hline & $(5.75)$ & $(5.95)$ & $(4.85)$ & $(4.60)$ \\
\hline \multirow{2}{*}{$\mathrm{T}_{9}$ Weed free check (hand weeding at 20 and 40 DAS ) } & 1.96 & 1.94 & 1.64 & 1.56 \\
\hline & (3.35) & $(3.25)$ & \begin{tabular}{|l|l|}
$(2.20)$ \\
\end{tabular} & $(1.95)$ \\
\hline \multirow{2}{*}{$\mathrm{T}_{10}$ Weedy check } & 3.73 & 4.01 & 3.66 & 3.60 \\
\hline & $(13.40)$ & $(15.60)$ & $(12.95)$ & $(12.45)$ \\
\hline SEm \pm & 0.04 & 0.08 & 0.06 & 0.06 \\
\hline $\mathrm{CD}(\mathrm{P}=0.05)$ & 0.14 & 0.023 & 0.18 & 0.18 \\
\hline
\end{tabular}

\subsubsection{Other weed species (No. $\mathrm{m}^{-2}$ )}

"At 30 DAS, however, the substantially lower density of other weed species was reported for weed-free treatment (hand weeding at 20 and 40 DAS) (T9) but was equivalent to the treatment of Pendimethalin 30 EC + Imazethapyr 2\% (Ready mix)@1,0 kg ha-1 PE + one hoeing at 30 DAS (T6), Topramezone 33.6 SC formulation (20.6 g a.i./ha) EPoE at 14 DAS $\left(\mathrm{T}_{1}\right)$, Pendimethalin $30 \mathrm{EC}$ formulation + Imazethapyr 2\% (Ready mix@1kg/ha) PE" fb Topramezone 33.6 SC (20.6 g a.i./ha) EPoE at $21 \mathrm{DAS}\left(\mathrm{T}_{7}\right)$, and Topramezone 33.6 $\mathrm{SC}$ formulation(25.7 g a.i./ha) $\mathrm{EPoE}$ at $14 \mathrm{DAS}\left(\mathrm{T}_{3}\right)$, respectively.
With respect to subsequent observation intervals "at 60,90 DAS and at harvest, the lower density of other weed species was reported under weed-free (hand weeding at 20 and 40 DAS) (T9), which, was statistically at par with the treatment of Pendimethalin 30 EC + Imazethapyr 2\% (Ready mix) @ 1.0 $\mathrm{kg} \mathrm{ha}^{-1} \mathrm{PE}+$ one hoeing at $\left.30 \mathrm{DAS}\left(\mathrm{T}_{6}\right)\right)$, Topramezone 33.6 $\mathrm{SC}$ formulation (20.6 g a.i./ha) EPoE at $14 \mathrm{DAS}\left(\mathrm{T}_{1}\right)$, Pendimethalin 30 EC formulation + Imazethapyr 2\% (Ready mix @ 1kg/ha) PE” fb Topramezone 33.6 SC (20.6 g a.i./ha) EPoE at 21 DAS $\left(\mathrm{T}_{7}\right)$, and Topramezone 33.6 SC formulation (25.7 g a.i./ha) EPoE at 14 DAS $\left(\mathrm{T}_{3}\right)$ The highest density was recorded under weedy check $\left(\mathrm{T}_{10}\right)$ (Table 5 .).

Table 5: Density of Other weeds in chickpea as influenced by different weed control measures

\begin{tabular}{|c|c|c|c|c|}
\hline \multirow{2}{*}{ Treatment } & \multicolumn{4}{|c|}{ Weed population $\left(\right.$ No.m $\left.^{-2}\right)$} \\
\hline & 30 DAS & 60 DAS & 90 DAS & t harvest \\
\hline \multirow{2}{*}{$\mathrm{T}_{1}$ Topramezone $33.6 \mathrm{SC}$ formulation (20.6 g a.i./ha) EPoE at $14 \mathrm{DAS}$} & 1.92 & 1.97 & 1.83 & 1.80 \\
\hline & $(3.20)$ & $(3.40)$ & $(2.85)$ & $(2.75)$ \\
\hline \multirow{2}{*}{$\mathrm{T}_{2}$ Topramezone $33.6 \mathrm{SC}$ formulation (20.6 g a.i./ha) EPoE at $21 \mathrm{DAS}$} & 2.09 & 2.13 & 2.02 & 1.96 \\
\hline & $(3.85)$ & $(4.05)$ & $(3.60)$ & $(3.35)$ \\
\hline \multirow{2}{*}{$\mathrm{T}_{3}$ Topramezone $33.6 \mathrm{SC}$ formulation (25.7 $\mathrm{g}$ a.i./ha) EPoE at $14 \mathrm{DAS}$} & 2.04 & 2.06 & 1.92 & 1.87 \\
\hline & $(3.65)$ & $(3.75)$ & $(3.20)$ & $(3.00)$ \\
\hline \multirow{2}{*}{$\mathrm{T}_{4}$ Topramezone $33.6 \mathrm{SC}$ formulation (25.7 g a.i./ha) EPoE at $21 \mathrm{DAS}$} & 2.07 & 2.14 & 2.04 & 1.97 \\
\hline & $(3.80)$ & $(4.10)$ & $(3.65)$ & $(3.40)$ \\
\hline \multirow{2}{*}{$\mathrm{T}_{5}$ Quizalofop-p-ethyl 5 EC (100 g a.i./ha) PoE at 25 DAS } & 2.11 & 2.18 & 2.13 & 2.01 \\
\hline & $(3.95)$ & $(4.25)$ & $(4.05)$ & $(3.55)$ \\
\hline \multirow{2}{*}{$\begin{array}{c}\text { T6 Pendimethalin } 30 \text { EC formulation + Imazethapyr 2\% (Ready mix @ 1kg/ha) PE + one Hoeing /hand } \\
\text { weeding at } 30 \text { DAS }\end{array}$} & 1.70 & 1.69 & 1.43 & 1.36 \\
\hline & $(2.40)$ & $(2.35)$ & $(1.55)$ & $(1.35)$ \\
\hline $\mathrm{T}_{7}$ Pendimethalin 30 EC formulation + Imazethapyr 2\% (Ready mix @ 1kg/ha) PE followed by & 1.92 & 2.01 & 1.90 & 1.85 \\
\hline
\end{tabular}




\begin{tabular}{|c|c|c|c|c|}
\hline Topramezone 33.6 SC (20.6 g a.i./ha) EPoE at $21 \mathrm{DAS}$ & $(3.25)$ & $(3.55)$ & $(3.10)$ & $(2.95)$ \\
\hline \multirow{2}{*}{$\begin{array}{c}\text { T }_{8} \text { Pendimethalin } 30 \text { EC formulation + Imazethapyr 2\% (Ready mix @ 1kg/ha) PE followed by } \\
\text { Topramezone 33.6 SC (25.7 g a.i./ha) EPoE at } 21 \text { DAS }\end{array}$} & 2.06 & 2.11 & 1.93 & 1.89 \\
\hline & $(3.75)$ & $(3.95)$ & $(3.25)$ & $(3.10)$ \\
\hline \multirow{2}{*}{$\mathrm{T}_{9}$ Weed free check (hand weeding at 20 and 40 DAS) } & 1.66 & 1.63 & 1.39 & 1.32 \\
\hline & $(2.25)$ & $(2.15)$ & $(1.45)$ & $(1.25)$ \\
\hline \multirow{2}{*}{$\mathrm{T}_{10}$ Weedy check } & 3.12 & 3.34 & 3.02 & 2.97 \\
\hline & $(9.25)$ & $(10.70)$ & $(8.65)$ & $(8.35)$ \\
\hline SEm \pm & 0.06 & 0.06 & 0.05 & 0.04 \\
\hline $\mathrm{CD}(\mathrm{P}=0.05)$ & 0.17 & 0.18 & 0.14 & 0.12 \\
\hline
\end{tabular}

\subsection{Total and species wise dry matter production of weeds $\left(\mathrm{g} \mathrm{m}^{-2}\right)$}

Different weed control treatments at 30, 60, 90 DAS have greatly affected the overall weed dry matter, and at harvest and data are provided in Table 6. Present us. Substantially higher dry matter was observed for weed species, namely Medicago denticulata, Chenopodium album Echinochloa colona and others under weed control (T10) relative to the rest of the trees.

"At 30 DAS, significantly lower total dry matter of weed species was recorded under the treatment of weed free (hand weeding at 20 and $40 \mathrm{DAS})\left(\mathrm{T}_{9}\right)$, however, lower total weed dry matter was recorded under of Pendimethalin $30 \mathrm{EC}+$ Imazethapyr 2\% (Ready mix) @ $1.0 \mathrm{~kg} \mathrm{ha}^{-1} \mathrm{PE}+$ one hoeing at 30 DAS $\left(\mathrm{T}_{6}\right)$, Topramezone 33.6 SC formulation $(20.6 \mathrm{~g}$ a.i./ha) EPoE at 14 DAS $\left(\mathrm{T}_{1}\right)$, Pendimethalin 30 EC formulation + Imazethapyr 2\% (Ready mix @ $1 \mathrm{~kg} / \mathrm{ha}$ ) PE $f b$ Topramezone 33.6 SC (20.6 g a.i./ha) EPoE at $21 \mathrm{DAS}\left(\mathrm{T}_{7}\right)$ and Topramezone 33.6 SC formulation(25.7 $\mathrm{g}$ a.i./ha) EPoE at 14 DAS $\left(T_{3}\right)$ as compared to weedy check $\left(T_{10}\right)$ and rest of the treatments. At later time interval of observed i.e. at 60, 90 DAS and at harvest, weed free (hand weeding at 20 and 40 DAS) $\left(T_{9}\right)$ resulted in significant reduction in total dry matter production of weeds which, was significantly superior over other treatments. However, among the herbicidal weed management treatments, significantly lower total weed dry matter was recorded under Pendimethalin 30 EC + Imazethapyr $2 \%$ (Ready mix) @ $1.0 \mathrm{~kg} \mathrm{ha}^{-1} \mathrm{PE}+$ one hoeing at $30 \mathrm{DAS}\left(\mathrm{T}_{6}\right)$, Topramezone $33.6 \mathrm{SC}$ formulation $(20.6 \mathrm{~g}$ a.i./ha) EPoE at 14 DAS $\left(\mathrm{T}_{1}\right)$, Pendimethalin 30 EC formulation + Imazethapyr 2\% (Ready mix @ 1kg/ha) PE" fb Topramezone 33.6 SC (20.6 g a.i./ha) EPoE at 21 DAS ( $\left.\mathrm{T}_{7}\right)$ and Topramezone 33.6 SC formulation (25.7 g a.i./ha) EPoE at $14 \mathrm{DAS}\left(\mathrm{T}_{3}\right)$ which were at par to each other at all the later stages of observation i.e. 60, 90 DAS and at harvest, and were superior over rest of the treatments. While highest dry matter production was noted under weedy check $\left(\mathrm{T}_{10}\right)$. These result are in accordance with the finding of Sharma (2009) ${ }^{[13]}$, Bhutada and Bhale (2013) ${ }^{[5]}$ and Chandrakar et al. (2015) ${ }^{[7]}$

Table 6: Total weeds dry weight of chickpea as influenced by different weed control measures

\begin{tabular}{|c|c|c|c|c|}
\hline Treatment & \multicolumn{4}{|c|}{ Weeds dry weight $\left(\mathrm{g} \mathrm{m}^{-2}\right)$} \\
\hline & 30 DAS & $60 \mathrm{DAS}$ & 90 DAS & it harvest \\
\hline \multirow{2}{*}{$\mathrm{T}_{1}$ Topramezone $33.6 \mathrm{SC}$ formulation(20.6 g a.i./ha) EPoE at $14 \mathrm{DAS}$} & 3.95 & 3.71 & 4.57 & 4.88 \\
\hline & $(15.17)$ & $(13.37)$ & $(20.41)$ & $(23.36)$ \\
\hline \multirow{2}{*}{$\mathrm{T}_{2}$ Topramezone $33.6 \mathrm{SC}$ formulation(20.6 g a.i./ha) EPoE at $21 \mathrm{DAS}$} & 4.13 & 4.67 & 4.95 & 5.26 \\
\hline & $(16.57)$ & $(21.37)$ & $(24.10)$ & $(27.26)$ \\
\hline \multirow{2}{*}{$\mathrm{T}_{3}$ Topramezone $33.6 \mathrm{SC}$ formulation(25.7 $\mathrm{g}$ a.i./ha) EPoE at $14 \mathrm{DAS}$} & 4.02 & 4.14 & 4.63 & 4.91 \\
\hline & $(15.72)$ & $(16.70)$ & $(20.93)$ & $(23.69)$ \\
\hline \multirow{2}{*}{$\mathrm{T}_{4}$ Topramezone $33.6 \mathrm{SC}$ formulation (25.7 $\mathrm{g}$ a.i./ha) EPoE at $21 \mathrm{DAS}$} & 4.25 & 4.71 & 5.04 & 5.30 \\
\hline & \begin{tabular}{|l|}
$(17.55)$ \\
\end{tabular} & $(21.70)$ & (24.93) & (27.65) \\
\hline \multirow{2}{*}{ T5 Quizalofop-p-ethyl 5 EC (100 g a.i./ha) PoE at 25 DAS } & 4.27 & 4.78 & 5.08 & 5.31 \\
\hline & $(17.80)$ & $(22.52)$ & $(25.41)$ & $(27.72)$ \\
\hline \multirow{2}{*}{$\begin{array}{c}\mathrm{T}_{6} \text { Pendimethalin } 30 \mathrm{EC} \text { formulation + Imazethapyr 2\% (Ready mix @ 1kg/ha) PE + one Hoeing /hand } \\
\text { weeding at } 30 \text { DAS }\end{array}$} & 3.57 & 3.14 & 3.48 & 3.75 \\
\hline & $(12.25)$ & $(9.14)$ & (11.64) & $(13.61)$ \\
\hline \multirow{2}{*}{$\begin{array}{c}\mathrm{T}_{7} \text { Pendimethalin } 30 \text { EC formulation + Imazethapyr 2\% (Ready mix @ 1kg/ha) PE followed by } \\
\text { Topramezone 33.6 SC (20.6 g a.i./ha) EPoE at } 21 \text { DAS }\end{array}$} & 4.00 & 4.08 & 4.60 & 4.90 \\
\hline & $(15.56)$ & $(16.31)$ & $(20.70)$ & $(23.57)$ \\
\hline \multirow{2}{*}{$\begin{array}{c}\text { T8 Pendimethalin } 30 \text { EC formulation + Imazethapyr 2\% (Ready mix @ 1kg/ha) PE followed by } \\
\text { Topramezone 33.6 SC (25.7 g a.i./ha) EPoE at } 21 \text { DAS }\end{array}$} & 4.12 & 4.23 & 4.67 & 5.00 \\
\hline & $(16.45)$ & $(17.54)$ & $(21.41)$ & $(24.62)$ \\
\hline \multirow{2}{*}{$\mathrm{T}_{9}$ Weed free check (hand weeding at 20 and 40 DAS) } & 3.26 & 2.92 & 3.23 & 3.50 \\
\hline & $(10.15)$ & $(8.06)$ & $(9.97)$ & $(11.81)$ \\
\hline \multirow{2}{*}{$\mathrm{T}_{10}$ Weedy check } & 5.25 & 6.57 & 7.08 & 7.33 \\
\hline & $(27.12)$ & (42.78) & $(49.80)$ & (53.33) \\
\hline SEm \pm & 0.10 & 0.16 & 0.14 & 0.13 \\
\hline $\mathrm{CD}(\mathrm{P}=0.05)$ & 0.29 & 0.48 & 0.43 & 0.39 \\
\hline
\end{tabular}

\subsection{Species wise dry matter production of weeds $\left(\mathrm{g} \mathrm{m}^{-2}\right)$ 3.4.1 Medicago denticulata $\left(\mathrm{g} \mathrm{m}^{-2}\right)$}

At 30 DAS, Medicago denticulata substantially lowest dry matter production was reported in weed-free care (hand weeding at 20 and 40 DAS) (T9), but it was on a par with care of Pendimethalin 30 EC + Imazethapyr 2\% (Ready mix) @ 1.0 $\mathrm{kg} \mathrm{ha}^{-1} \mathrm{PE}+$ one hoeing at $30 \mathrm{DAS}\left(\mathrm{T}_{6}\right)$, Topramezone 33.6 $\mathrm{SC}$ formulation (20.6 $\mathrm{g}$ a.i./ha) EPoE at $14 \mathrm{DAS}\left(\mathrm{T}_{1}\right)$, Pendimethalin 30 EC formulation + Imazethapyr 2\% (Ready mix @ 1kg/ha) PE fb Topramezone 33.6 SC (20.6 g a.i./ha)
EPoE at 21 DAS $\left(\mathrm{T}_{7}\right)$ and Topramezone $33.6 \mathrm{SC}$ formulation(25.7 $\mathrm{g}$ a.i./ha) EPoE at $14 \mathrm{DAS}\left(\mathrm{T}_{3}\right)$. (Table 7). At 60, 90 DAS as well as at harvest, the minimum production of dry weeds was recorded under weed-free (hand weeding at 20 and 40 DAS) (T9), which was substantially superior to other treatments. Among the herbicides, significantly lower dry matter production was observed in Pendimethalin $30 \mathrm{EC}$ + Imazethapyr 2\% (Ready mix) @ $1.0 \mathrm{~kg} \mathrm{ha}^{-1} \mathrm{PE}+$ one hoeing at $30 \mathrm{DAS}\left(\mathrm{T}_{6}\right)$, Topramezone 33.6 SC formulation (20.6 g a.i./ha) EPoE at 14 DAS $\left(\mathrm{T}_{1}\right)$, Pendimethalin 30 EC 
formulation + Imazethapyr 2\% (Ready mix @ 1kg/ha) PE $f b$ Topramezone 33.6 SC (20.6 g a.i./ha) EPoE at $21 \mathrm{DAS}\left(\mathrm{T}_{7}\right)$ and Topramezone 33.6 SC formulation(25.7 $\mathrm{g}$ a.i./ha) EPoE at
14 DAS $\left(\mathrm{T}_{3}\right)$. The maximum dry weight of Medicago denticulata was recorded weedy check $\left(\mathrm{T}_{10}\right)$.

Table 7: Dry matter of Medicago denticulata in chickpea as influenced by different weed control measures

\begin{tabular}{|c|c|c|c|c|}
\hline \multirow[t]{2}{*}{ Treatment } & \multicolumn{4}{|c|}{ Dry matter $\left(\mathrm{g} \mathrm{m}^{-2}\right)$} \\
\hline & \multicolumn{4}{|c|}{30 DAS 60 DAS 90 DASAt harves } \\
\hline \multirow[t]{2}{*}{$\mathrm{T}_{1}$ Topramezone $33.6 \mathrm{SC}$ formulation(20.6 g a.i./ha) EPoE at $14 \mathrm{DAS}$} & 2.84 & 2.67 & 3.27 & 3.49 \\
\hline & $(7.64)$ & $(6.68)$ & $(10.21)$ & $(11.68)$ \\
\hline \multirow[t]{2}{*}{$\mathrm{T}_{2}$ Topramezone $33.6 \mathrm{SC}$ formulation(20.6 g a.i./ha) EPoE at $21 \mathrm{DAS}$} & 2.96 & 3.34 & 3.54 & 3.76 \\
\hline & $(8.28)$ & $(10.69)$ & $(12.06)$ & $(13.63)$ \\
\hline \multirow[t]{2}{*}{$\mathrm{T}_{3}$ Topramezone $33.6 \mathrm{SC}$ formulation(25.7 g a.i./ha) EPoE at $14 \mathrm{DAS}$} & 2.89 & 2.97 & 3.31 & 3.51 \\
\hline & $(7.87)$ & $(8.36)$ & $(10.47)$ & $(11.85)$ \\
\hline \multirow{2}{*}{$\mathrm{T}_{4}$ Topramezone $33.6 \mathrm{SC}$ formulation (25.7 $\mathrm{g}$ a.i./ha) EPoE at $21 \mathrm{DAS}$} & 3.05 & 3.37 & 3.60 & 3.78 \\
\hline & $(8.78)$ & $(10.86)$ & $(12.47)$ & $(13.82)$ \\
\hline \multirow{2}{*}{$\mathrm{T}_{5}$ Quizalofop-p-ethyl 5 EC (100 g a.i./ha) PoE at $25 \mathrm{DAS}$} & 3.06 & 3.42 & 3.63 & 3.78 \\
\hline & $(8.91)$ & $(11.28)$ & $(12.71)$ & $(13.85)$ \\
\hline \multirow{2}{*}{$\begin{array}{l}\text { T6 Pendimethalin } 30 \text { EC formulation + Imazethapyr } 2 \% \text { (Ready mix @ 1kg/ha) PE + one Hoeing /hand } \\
\text { weeding at } 30 \text { DAS }\end{array}$} & 2.57 & 2.27 & 2.51 & 2.70 \\
\hline & $(6.13)$ & $(4.68)$ & $(5.82)$ & $(6.81)$ \\
\hline \multirow{2}{*}{$\begin{array}{c}\text { T7 Pendimethalin } 30 \text { EC formulation + Imazethapyr 2\% (Ready mix @ 1kg/ha) PE followed by } \\
\text { Topramezone 33.6 SC (20.6 g a.i./ha) EPoE at } 21 \text { DAS }\end{array}$} & 2.87 & 2.93 & 3.29 & 3.50 \\
\hline & \begin{tabular}{|l}
$(7.77)$ \\
\end{tabular} & $(8.16)$ & $(10.35)$ & (11.79) \\
\hline \multirow{2}{*}{$\begin{array}{c}\left.\mathrm{T}_{8} \text { Pendimethalin } 30 \text { EC formulation + Imazethapyr 2\% (Ready mix @ } 1 \mathrm{~kg} / \mathrm{ha}\right) \text { PE followed by } \\
\text { Topramezone 33.6 SC (25.7 g a.i./ha) EPoE at } 21 \text { DAS }\end{array}$} & 2.95 & 3.03 & 3.34 & 3.57 \\
\hline & $(8.23)$ & $(8.78)$ & $(10.71)$ & $(12.25)$ \\
\hline \multirow{2}{*}{$\mathrm{T}_{9}$ Weed free check (hand weeding at 20 and 40 DAS ) } & 2.36 & 2.13 & 2.34 & 2.52 \\
\hline & $(5.08)$ & $(4.03)$ & $(4.98)$ & $(5.90)$ \\
\hline \multirow{2}{*}{$\mathrm{T}_{10}$ Weedy check } & 4.06 & 4.67 & 5.03 & 5.21 \\
\hline & $(16.04)$ & $(21.39)$ & $(24.90)$ & $(26.67)$ \\
\hline SEm \pm & 0.08 & 0.11 & 0.10 & 0.10 \\
\hline $\mathrm{CD}(\mathrm{P}=0.05)$ & 0.23 & 0.33 & 0.30 & 0.29 \\
\hline
\end{tabular}

\subsubsection{Echinochloa colona $\left(\mathrm{g} \mathrm{m}^{-2}\right)$}

At 30 DAS, the dry matter development of Echinochloa colona was substantially lowest under weed-free treatment (hand weeding at 20 and 40 DAS) (T9), but was on par with treatment of Pendimethalin $30 \mathrm{EC}+$ Imazethapyr 2\% (Ready mix) @ $1.0 \mathrm{~kg} \mathrm{ha}^{-1} \mathrm{PE}+$ one hoeing at $30 \mathrm{DAS}\left(\mathrm{T}_{6}\right)$, Topramezone 33.6 SC formulation (20.6 g a.i./ha) EPoE at 14 DAS $\left(\mathrm{T}_{1}\right)$, Pendimethalin $30 \mathrm{EC}$ formulation + Imazethapyr 2\% (Ready mix @ 1kg/ha) PE fb Topramezone 33.6 SC (20.6 $\mathrm{g}$ a.i./ha) EPoE at $21 \mathrm{DAS}\left(\mathrm{T}_{7}\right)$ and Topramezone $33.6 \mathrm{SC}$ formulation (25.7 $\mathrm{g}$ a.i./ha) EPoE at $14 \mathrm{DAS}\left(\mathrm{T}_{3}\right)$ respectively (Table 8).

Substantially lowest "dry weight was noted under weed free (hand weeding at 20 and 40 DAS) (T9) at 60, 90 DAS and at harvest, which was clearly better over other treatments. The maximum dry weight of Echinochloa colona was recorded under weedy check $\left(\mathrm{T}_{10}\right)$. Among the herbicidal weed management, significantly lowest dry weight was observed in Pendimethalin $30 \mathrm{EC}$ + Imazethapyr 2\% (Ready mix) @ 1.0 $\mathrm{kg} \mathrm{ha}^{-1} \mathrm{PE}+$ one hoeing at $30 \mathrm{DAS}\left(\mathrm{T}_{6}\right)$, Topramezone 33.6 $\mathrm{SC}$ formulation (20.6 g a.i./ha) EPoE at $14 \mathrm{DAS}\left(\mathrm{T}_{1}\right)$, Pendimethalin 30 EC formulation + Imazethapyr 2\% (Ready mix @ 1kg/ha) PE $f b$ Topramezone 33.6 SC (20.6 g a.i./ha) EPoE at 21 DAS $\left(\mathrm{T}_{7}\right)$ and Topramezone $33.6 \mathrm{SC}$ formulation(25.7 $\mathrm{g}$ a.i./ha) $\mathrm{EPoE}$ at $14 \mathrm{DAS}\left(\mathrm{T}_{3}\right)$ and both were at par to each other and significantly superior over rest of the treatments.

Table 8: Dry matter of Echinochloa colona in chickpea as influenced by different weed control measures

\begin{tabular}{|c|c|c|c|c|}
\hline \multirow[t]{2}{*}{ Treatment } & \multicolumn{4}{|c|}{ Dry matter $\left(\mathrm{g} \mathrm{m}^{-2}\right)$} \\
\hline & 30 DAS & $60 \mathrm{DAS}$ & $90 \mathrm{DAS}$ & t harvest \\
\hline \multirow{2}{*}{$\mathrm{T}_{1}$ Topramezone $33.6 \mathrm{SC}$ formulation (20.6 g a.i./ha) EPoE at $14 \mathrm{DAS}$} & 2.07 & 1.96 & 2.36 & 2.52 \\
\hline & $(3.82)$ & $(3.34)$ & $(5.10)$ & $(5.84)$ \\
\hline \multirow{2}{*}{$\mathrm{T}_{2}$ Topramezone $33.6 \mathrm{SC}$ formulation (20.6 g a.i./ha) EPoE at $21 \mathrm{DAS}$} & 2.15 & 2.42 & 2.55 & 2.70 \\
\hline & $(4.14)$ & $(5.34)$ & $(6.03)$ & $(6.82)$ \\
\hline \multirow{2}{*}{$\mathrm{T}_{3}$ Topramezone $33.6 \mathrm{SC}$ formulation (25.7 g a.i./ha) EPoE at $14 \mathrm{DAS}$} & 2.11 & 2.16 & 2.39 & 2.53 \\
\hline & $(3.95)$ & $\begin{array}{l}(4.18) \\
\end{array}$ & $(5.23)$ & $\begin{array}{l}(5.92) \\
\end{array}$ \\
\hline \multirow{2}{*}{$\mathrm{T}_{4}$ Topramezone $33.6 \mathrm{SC}$ formulation ( $25.7 \mathrm{~g}$ a.i./ha) EPoE at $21 \mathrm{DAS}$} & 2.21 & 2.43 & 2.59 & 2.72 \\
\hline & $(4.39)$ & $(5.43)$ & $(6.23)$ & $(6.92)$ \\
\hline \multirow{2}{*}{ T5 Quizalofop-p-ethyl 5 EC (100 g a.i./ha) PoE at 25 DAS } & 2.22 & 2.47 & 2.61 & 2.72 \\
\hline & \begin{tabular}{|l|}
$(4.45)$ \\
\end{tabular} & $(5.64)$ & $(6.35)$ & $(6.94)$ \\
\hline \multirow{2}{*}{$\begin{array}{c}\mathrm{T}_{6} \text { Pendimethalin } 30 \mathrm{EC} \text { formulation + Imazethapyr } 2 \% \text { (Ready mix @ 1 kg/ha) PE + one Hoeing /hand } \\
\text { weeding at } 30 \text { DAS }\end{array}$} & 1.89 & 1.68 & 1.84 & 1.97 \\
\hline & \begin{tabular}{|l|}
$(3.06)$ \\
\end{tabular} & $(2.34)$ & \begin{tabular}{|l|}
$(2.91)$ \\
\end{tabular} & $(3.40)$ \\
\hline \multirow{2}{*}{$\begin{array}{c}\text { T }_{7} \text { Pendimethalin } 30 \text { EC formulation + Imazethapyr 2\% (Ready mix @ 1kg/ha) PE followed by } \\
\text { Topramezone 33.6 SC (20.6 g a.i./ha) EPoE at } 21 \text { DAS }\end{array}$} & 2.10 & 2.13 & 2.38 & 2.53 \\
\hline & (3.93) & $(4.08)$ & $(5.17)$ & (5.89) \\
\hline \multirow{2}{*}{$\begin{array}{c}\text { T8 Pendimethalin } 30 \text { EC formulation + Imazethapyr 2\% (Ready mix @ 1kg/ha) PE followed by } \\
\text { Topramezone 33.6 SC (25.7 g a.i./ha) EPoE at } 21 \text { DAS }\end{array}$} & 2.15 & 2.20 & 2.41 & 2.58 \\
\hline & (4.11) & $(4.39)$ & $(5.35)$ & $(6.19)$ \\
\hline \multirow{2}{*}{$\mathrm{T}_{9}$ Weed free check (hand weeding at 20 and 40 DAS ) } & 1.74 & 1.58 & 1.73 & 1.85 \\
\hline & $(2.54)$ & $(2.01)$ & \begin{tabular}{|l}
$(2.49)$ \\
\end{tabular} & $(2.95)$ \\
\hline $\mathrm{T}_{10}$ Weedy check & 1.89 & 3.34 & 3.59 & 3.72 \\
\hline
\end{tabular}




\begin{tabular}{|c|c|c|c|c|}
\hline & $(3.06)$ & $(10.70)$ & $(12.45)$ & $(13.33)$ \\
\hline SEm \pm & 0.05 & 0.08 & 0.07 & 0.07 \\
\hline $\mathrm{CD}(\mathrm{P}=0.05)$ & 0.15 & 0.23 & 0.20 & 0.20 \\
\hline
\end{tabular}

\subsubsection{Chenopodium album $\left(\mathrm{g} \mathrm{m}^{-2}\right)$}

At 30 DAS, significantly lower dry matter production of Chenopodium album was observed in the treatment of weed free (hand weeding at 20 and $40 \mathrm{DAS})\left(\mathrm{T}_{9}\right)$, however, it was at par to the treatment of Pendimethalin $30 \mathrm{EC}+$ Imazethapyr $2 \%$ (Ready mix) @ $1.0 \mathrm{~kg} \mathrm{ha}^{-1} \mathrm{PE}+$ one hoeing at $30 \mathrm{DAS}$ ( $\mathrm{T}_{6}$ ), Topramezone 33.6 SC formulation (20.6 g a.i./ha) EPoE at 14 DAS $\left(\mathrm{T}_{1}\right)$, Pendimethalin $30 \mathrm{EC}$ formulation + Imazethapyr 2\% (Ready mix @ 1kg/ha) PE $f b$ Topramezone 33.6 SC (20.6 g a.i./ha) EPoE at 21 DAS $\left(\mathrm{T}_{7}\right)$ and Topramezone 33.6 SC formulation(25.7 g a.i./ha) EPoE at 14 DAS $\left(\mathrm{T}_{3}\right)$ respectively (Table 9$)$.

"At 60, 90 DAS and at harvest, significantly lower dry matter production was recorded under weed free (hand weeding at 20 and $40 \mathrm{DAS})\left(\mathrm{T}_{9}\right)$, than weedy check $\left(\mathrm{T}_{10}\right)$. The significantly maximam dry matter production of Chenopodium album was under weedy check $\left(\mathrm{T}_{10}\right)$. Among the herbicidal treatments, at At 60, 90 DAS and at harvest, treatment of Pendimethalin 30 $\mathrm{EC}+$ Imazethapyr 2\% (Ready mix) @ $1.0 \mathrm{~kg} \mathrm{ha}^{-1} \mathrm{PE}+$ one hoeing at 30 DAS $\left(\mathrm{T}_{6}\right)$, Topramezone $33.6 \mathrm{SC}$ formulation (20.6 g a.i./ha) EPoE at $14 \mathrm{DAS}\left(\mathrm{T}_{1}\right)$, Pendimethalin $30 \mathrm{EC}$ formulation + Imazethapyr 2\% (Ready mix @ $1 \mathrm{~kg} / \mathrm{ha}$ ) PE $f b$ Topramezone 33.6 SC (20.6 g a.i./ha) EPoE at $21 \mathrm{DAS}\left(\mathrm{T}_{7}\right)$ and Topramezone 33.6 SC formulation(25.7 $\mathrm{g}$ a.i./ha) EPoE at 14 DAS $\left(\mathrm{T}_{3}\right)$ were at par to the weed free (hand weeding at 20 and $40 \mathrm{DAS})\left(\mathrm{T}_{9}\right)$, respectively, but, superior over rest of the treatments and were" significantly effective in reducing the dry matter production of Chenopodium album than rest of the herbicidal treatments including weedy check $\left(\mathrm{T}_{10}\right)$.

Table 9: Dry matter of Chenopodium album in chickpea as influenced by different weed control measures

\begin{tabular}{|c|c|c|c|c|}
\hline \multirow[t]{2}{*}{ Treatment } & \multicolumn{4}{|c|}{ Dry matter $\left(\mathrm{g} \mathrm{m}^{-2}\right)$} \\
\hline & 30 DAS & 60 DAS & 90 DAS & It harvest \\
\hline \multirow{2}{*}{$\mathrm{T}_{1}$ Topramezone $33.6 \mathrm{SC}$ formulation (20.6 g a.i./ha) EPoE at $14 \mathrm{DAS}$} & 1.64 & 1.58 & 1.89 & 2.00 \\
\hline & $(2.19)$ & $(2.01)$ & $(3.06)$ & $(3.50)$ \\
\hline \multirow{2}{*}{$\mathrm{T}_{2}$ Topramezone $33.6 \mathrm{SC}$ formulation (20.6 g a.i./ha) EPoE at $21 \mathrm{DAS}$} & 1.73 & 1.92 & 2.03 & 2.14 \\
\hline & $(2.48)$ & $(3.20)$ & $(3.61)$ & $(4.09)$ \\
\hline \multirow{2}{*}{$\mathrm{T}_{3}$ Topramezone $33.6 \mathrm{SC}$ formulation $(25.7 \mathrm{~g}$ a.i./ha) EPoE at $14 \mathrm{DAS}$} & 1.68 & 1.73 & 1.91 & 2.01 \\
\hline & $(2.33)$ & $(2.50)$ & $(3.14)$ & $(3.55)$ \\
\hline \multirow{2}{*}{$\mathrm{T}_{4}$ Topramezone $33.6 \mathrm{SC}$ formulation ( $25.7 \mathrm{~g}$ a.i./ha) EPoE at $21 \mathrm{DAS}$} & 1.77 & 1.94 & 2.06 & 2.16 \\
\hline & $(2.63)$ & $(3.25)$ & $(3.74)$ & $(4.15)$ \\
\hline \multirow{2}{*}{$\mathrm{T}_{5}$ Quizalofop-p-ethyl 5 EC (100 g a.i./ha) PoE at 25 DAS } & 1.78 & 1.96 & 2.07 & 2.16 \\
\hline & $(2.67)$ & $(3.36)$ & \begin{tabular}{|l|}
$(3.81)$ \\
\end{tabular} & $(4.16)$ \\
\hline \multirow{2}{*}{$\begin{array}{c}\mathrm{T}_{6} \text { Pendimethalin } 30 \mathrm{EC} \text { formulation + Imazethapyr } 2 \% \text { (Ready mix @ 1kg/ha) PE + one Hoeing /hand weeding at } \\
\text { 30 DAS }\end{array}$} & 1.53 & 1.40 & 1.50 & 1.59 \\
\hline & $(1.84)$ & $(1.45)$ & \begin{tabular}{|l|}
$(1.75)$ \\
\end{tabular} & $(2.04)$ \\
\hline \multirow{2}{*}{$\begin{array}{l}\left.\mathrm{T}_{7} \text { Pendimethalin } 30 \mathrm{EC} \text { formulation + Imazethapyr 2\% (Ready mix @ } 1 \mathrm{~kg} / \mathrm{ha}\right) \text { PE followed by Topramezone } 33.6 \\
\text { SC (20.6 g a.i./ha) EPoE at } 21 \mathrm{DAS}\end{array}$} & 1.66 & 1.71 & 1.90 & 2.01 \\
\hline & \begin{tabular}{|l}
$(2.29)$ \\
\end{tabular} & $(2.44)$ & \begin{tabular}{|l|}
$(3.10)$ \\
\end{tabular} & (3.53) \\
\hline \multirow{2}{*}{$\begin{array}{c}\left.\mathrm{T}_{8} \text { Pendimethalin } 30 \mathrm{EC} \text { formulation + Imazethapyr } 2 \% \text { (Ready mix @ } 1 \mathrm{~kg} / \mathrm{ha}\right) \text { PE followed by Topramezone } 33.6 \\
\text { SC (25.7 g a.i./ha) EPoE at } 21 \text { DAS }\end{array}$} & 1.72 & 1.76 & 1.92 & 2.05 \\
\hline & $(2.47)$ & $(2.63)$ & \begin{tabular}{|l|}
$(3.21)$ \\
\end{tabular} & $(3.71)$ \\
\hline \multirow{2}{*}{$\mathrm{T}_{9}$ Weed free check (hand weeding at 20 and 40 DAS ) } & 1.42 & 1.31 & 1.41 & 1.50 \\
\hline & $(1.52)$ & $(1.21)$ & \begin{tabular}{|l}
$(1.50)$ \\
\end{tabular} & $(1.77)$ \\
\hline \multirow{2}{*}{$\mathrm{T}_{10}$ Weedy check } & 2.30 & 2.63 & 2.82 & 2.91 \\
\hline & $(4.81)$ & $(6.42)$ & \begin{tabular}{|l|}
$(7.47)$ \\
\end{tabular} & $(8.00)$ \\
\hline SEm \pm & 0.04 & 0.05 & 0.05 & 0.05 \\
\hline $\mathrm{CD}(\mathrm{P}=0.05)$ & 0.11 & 0.17 & 0.15 & 0.15 \\
\hline
\end{tabular}

\subsubsection{Other weed species $\left(\mathrm{gm}^{-2}\right)$}

"At 30 DAS, weed free (hand weeding at 20 and 40 DAS) $\left(\mathrm{T}_{9}\right)$, produced lower dry matter of other weed species, however, it was at par to the treatment of of Pendimethalin 30 EC + Imazethapyr 2\% (Ready mix) @ $1.0 \mathrm{~kg} \mathrm{ha}^{-1} \mathrm{PE}+$ one hoeing at $30 \mathrm{DAS}\left(\mathrm{T}_{6}\right)$, Topramezone $33.6 \mathrm{SC}$ formulation (20.6 g a.i./ha) EPoE at 14 DAS ( $\left.\mathrm{T}_{1}\right)$, Pendimethalin $30 \mathrm{EC}$ formulation + Imazethapyr 2\% (Ready mix @ 1kg/ha) PE" $f b$ Topramezone 33.6 SC (20.6 g a.i./ha) EPoE at $21 \mathrm{DAS}\left(\mathrm{T}_{7}\right)$ and Topramezone 33.6 SC formulation (25.7 $\mathrm{g}$ a.i./ha) EPoE at $14 \mathrm{DAS}\left(\mathrm{T}_{3}\right)$ respectively.

With respect to later observation intervals, i.e. "at 60, 90 DAS and at harvest, the amount of minimum dry matter of other

Table 10: Dry matter of other weed species in chickpea as influenced by different weed control measures

\begin{tabular}{|c|c|c|c|c|}
\hline \multirow[t]{2}{*}{ Treatment } & \multicolumn{4}{|c|}{ Dry matter $\left(\mathrm{g} \mathrm{m}^{-2}\right)$} \\
\hline & \begin{tabular}{|c|} 
30 \\
DAS \\
\end{tabular} & $\begin{array}{c}60 \\
\text { DAS } \\
\end{array}$ & $\begin{array}{c}90 \\
\text { DAS } \\
\end{array}$ & $\begin{array}{c}\text { At } \\
\text { harvest }\end{array}$ \\
\hline \multirow{2}{*}{$\mathrm{T}_{1}$ Topramezone $33.6 \mathrm{SC}$ formulation(20.6 g a.i./ha) EPoE at $14 \mathrm{DAS}$} & 1.42 & 1.35 & 1.59 & 1.68 \\
\hline & $\begin{array}{l}(1.53) \\
\end{array}$ & $(1.34)$ & (2.04) & $(2.34)$ \\
\hline \multirow{2}{*}{$\mathrm{T}_{2}$ Topramezone $33.6 \mathrm{SC}$ formulation(20.6 g a.i./ha) EPoE at $21 \mathrm{DAS}$} & 1.47 & 1.62 & 1.70 & 1.79 \\
\hline & $(1.66)$ & $(2.14)$ & $(2.41)$ & $(2.72)$ \\
\hline
\end{tabular}

weed species was reported under weed-free (hand weeding at 20 and 40 DAS) (T9), which was statistically equivalent to treatment of Pendimethalin $30 \mathrm{EC}+$ Imazethapyr 2\% (Ready mix) @ $1.0 \mathrm{~kg} \mathrm{ha}^{-1} \mathrm{PE}+$ one hoeing at $30 \mathrm{DAS}\left(\mathrm{T}_{6}\right)$, Topramezone 33.6 SC formulation (20.6 g a.i./ha) EPoE at 14 DAS $\left(\mathrm{T}_{1}\right)$, Pendimethalin 30 EC formulation + Imazethapyr 2\% (Ready mix @ 1kg/ha) PE fb Topramezone 33.6 SC (20.6 $\mathrm{g}$ a.i./ha) EPoE at $21 \mathrm{DAS}\left(\mathrm{T}_{7}\right)$ and Topramezone $33.6 \mathrm{SC}$ formulation (25.7 $\mathrm{g}$ a.i./ha) EPoE at $14 \mathrm{DAS}\left(\mathrm{T}_{3}\right)$ as compared to weedy check $\left(\mathrm{T}_{10}\right)$ and other treatments. The low dry matter production was recorded under weedy check $\left(T_{10}\right)$ (Table 10). 


\begin{tabular}{|c|c|c|c|c|}
\hline \multirow{2}{*}{$\mathrm{T}_{3}$ Topramezone $33.6 \mathrm{SC}$ formulation(25.7 g a.i./ha) EPoE at $14 \mathrm{DAS}$} & 1.44 & 1.47 & 1.61 & 1.69 \\
\hline & $(1.58)$ & $(1.67)$ & $(2.09)$ & $(2.37)$ \\
\hline \multirow{2}{*}{$\mathrm{T}_{4}$ Topramezone $33.6 \mathrm{SC}$ formulation (25.7 $\mathrm{g}$ a.i./ha) EPoE at $21 \mathrm{DAS}$} & 1.50 & 1.63 & 1.73 & 1.81 \\
\hline & \begin{tabular}{|l|}
$(1.75)$ \\
\end{tabular} & $(2.17)$ & $(2.49)$ & $(2.77)$ \\
\hline \multirow{2}{*}{$\mathrm{T}_{5}$ Quizalofop-p-ethyl 5 EC (100 g a.i./ha) PoE at 25 DAS } & 1.51 & 1.65 & 1.74 & 1.81 \\
\hline & $(1.78)$ & $(2.25)$ & $(2.54)$ & $(2.77)$ \\
\hline \multirow{2}{*}{$\begin{array}{c}\text { T6 Pendimethalin } 30 \text { EC formulation + Imazethapyr 2\% (Ready mix @ 1 kg/ha) PE + one Hoeing /hand } \\
\text { weeding at } 30 \text { DAS }\end{array}$} & 1.31 & 1.20 & 1.29 & 1.36 \\
\hline & $(1.23)$ & $(0.94)$ & $(1.16)$ & $(1.36)$ \\
\hline \multirow{2}{*}{$\begin{array}{l}\mathrm{T}_{7} \text { Pendimethalin } 30 \mathrm{EC} \text { formulation + Imazethapyr 2\% (Ready mix @ 1kg/ha) PE followed by Topramezone } \\
33.6 \mathrm{SC}(20.6 \mathrm{~g} \text { a.i./ha) EPoE at } 21 \mathrm{DAS}\end{array}$} & 1.44 & 1.45 & 1.60 & 1.69 \\
\hline & \begin{tabular}{|l}
$(1.57)$ \\
\end{tabular} & (1.63) & $(2.07)$ & $(2.35)$ \\
\hline \multirow{2}{*}{$\begin{array}{l}\text { T8 Pendimethalin } 30 \text { EC formulation + Imazethapyr 2\% (Ready mix @ 1kg/ha) PE followed by Topramezone } \\
33.6 \mathrm{SC}(25.7 \mathrm{~g} \text { a.i./ha) EPoE at } 21 \mathrm{DAS}\end{array}$} & 1.46 & 1.50 & 1.62 & 1.72 \\
\hline & $(1.64)$ & $(1.75)$ & $(2.14)$ & $(2.47)$ \\
\hline \multirow{2}{*}{$\mathrm{T}_{9}$ Weed free check (hand weeding at 20 and 40 DAS ) } & 1.23 & 1.14 & 1.22 & 1.29 \\
\hline & $(1.02)$ & $(0.81)$ & $(1.00)$ & $(1.18)$ \\
\hline \multirow{2}{*}{$\mathrm{T}_{10}$ Weedy check } & 1.92 & 2.18 & 2.34 & 2.41 \\
\hline & $(3.21)$ & $(4.28)$ & $(4.98)$ & $(5.33)$ \\
\hline SEm \pm & 0.03 & 0.04 & 0.04 & 0.04 \\
\hline $\mathrm{CD}(\mathrm{P}=0.05)$ & 0.09 & 0.13 & 0.12 & 0.12 \\
\hline
\end{tabular}

\subsubsection{Weed control efficiency (WCE)}

"Weed control efficiency of different weed at 30, 60, 90 DAS and at harvest are Table 11. The data reflect the highest weed control efficiency was recorded under weed free (hand weeding at 20 and 40 DAS) $\left(\mathrm{T}_{9}\right) f b$ Pendimethalin $30 \mathrm{EC}+$ Imazethapyr 2\% (Ready mix) @ $1.0 \mathrm{~kg} \mathrm{ha}^{-1} \mathrm{PE}+$ one hoeing at $30 \mathrm{DAS}\left(\mathrm{T}_{6}\right)$, Topramezone $33.6 \mathrm{SC}$ formulation $(20.6 \mathrm{~g}$ a.i./ha) $\mathrm{EPoE}$ at $14 \mathrm{DAS}\left(\mathrm{T}_{1}\right)$, Pendimethalin $30 \mathrm{EC}$ formulation + Imazethapyr 2\% (Ready mix @ 1kg/ha) PE" fb
Topramezone 33.6 SC (20.6 g a.i./ha) EPoE at $21 \mathrm{DAS}\left(\mathrm{T}_{7}\right)$ and Topramezone 33.6 SC formulation(25.7 g a.i./ha) EPoE at 14 DAS $\left(T_{3}\right)$. This May well be attributed to decreased weed density and dry weight with a pre-emergence and PoE application to some degree of Pendimethali + Imazehyapyr and Topramezone. These result are in accordance with the finding of Ali et al. (2013), Bhutada and Bhale (2013), Kour et al. (2014) ${ }^{[8]}$ and Rathod et al. (2017).

Table 11: Weed control efficiency of chickpea as influenced by different weed control measures

\begin{tabular}{|c|c|c|c|c|}
\hline \multirow{2}{*}{ Treatment } & \multicolumn{4}{|c|}{\begin{tabular}{|l} 
Weed control efficiency (\%) \\
\end{tabular}} \\
\hline & 30 DA & 60 DA & $90 \mathrm{DA}$ & tharvest \\
\hline $\mathrm{T}_{1}$ Topramezone $33.6 \mathrm{SC}$ formulation (20.6 $\mathrm{g}$ a.i./ha) EPoE at $14 \mathrm{DAS}$ & 44.20 & 68.16 & 58.46 & 55.75 \\
\hline $\mathrm{T}_{2}$ Topramezone $33.6 \mathrm{SC}$ formulation (20.6 g a.i./ha)E PoE at $21 \mathrm{DAS}$ & 38.51 & 48.81 & 50.60 & 48.05 \\
\hline $\mathrm{T}_{3}$ Topramezone $33.6 \mathrm{SC}$ formulation $(25.7 \mathrm{~g}$ a.i./ha)E PoE at $14 \mathrm{DAS}$ & 41.82 & 60.05 & 57.37 & 54.95 \\
\hline $\mathrm{T}_{4}$ Topramezone $33.6 \mathrm{SC}$ formulation ( $25.7 \mathrm{~g}$ a.i./ha) EPoE at $21 \mathrm{DAS}$ & 34.85 & 48.18 & 49.25 & 47.70 \\
\hline$T_{5}$ Quizalofop-p-ethyl 5 EC (100 g a.i./ha) PoE at 25 DAS & 34.07 & 47.02 & 48.76 & 47.55 \\
\hline $\begin{array}{l}\mathrm{T}_{6} \text { Pendimethalin } 30 \mathrm{EC} \text { formulation + Imazethapyr } 2 \% \text { (Ready mix @ 1kg/ha) PE + } \\
\text { one Hoeing /hand weeding at } 30 \text { DAS }\end{array}$ & 54.30 & 77.94 & 76.68 & 74.34 \\
\hline $\begin{array}{l}\text { T7 Pendimethalin } 30 \mathrm{EC} \text { formulation + Imazethapyr 2\% (Ready mix @ 1kg/ha) PE } f b \text { Topramezone } 33.6 \mathrm{SC} \\
\text { (20.6 g a.i./ha) EPoE at } 21 \text { DAS }\end{array}$ & 42.48 & 62.20 & 58.43 & 55.84 \\
\hline $\begin{array}{l}\text { T8 Pendimethalin } 30 \text { EC formulation + Imazethapyr 2\% (Ready mix @ 1 kg/ha) PE } \\
\text { followed by Topramezone } 33.6 \mathrm{SC} \text { ( } 25.7 \mathrm{~g} \text { a.i./ha) EPoE at } 21 \mathrm{DAS}\end{array}$ & 39.15 & 58.84 & 56.89 & 53.64 \\
\hline$T_{9}$ Weed free check (hand weeding at 20 and 40 DAS ) & 62.38 & 80.98 & 79.79 & 77.63 \\
\hline $\mathrm{T}_{10}$ Weedy check & 0.00 & 0.00 & 0.00 & 0.00 \\
\hline SEm \pm & 2.97 & 3.42 & 2.84 & 2.47 \\
\hline $\mathrm{CD}(\mathrm{P}=0.05)$ & 8.64 & 9.94 & 8.25 & 7.17 \\
\hline
\end{tabular}

\section{Conclusions}

Significantly lower total and species wise "weed density and dry matter of weed species and total weed index were recorded under weed free" (hand weeding at 20 and 40 DAS). However, it was at par to the treatment of "Pendimethalin 30 EC + Imazethapyr 2\% (Ready mix) @ $1.0 \mathrm{~kg} \mathrm{ha}^{-1} \mathrm{PE}+$ one hoeing at 30 DAS", Topramezone 33.6 SC formulation (20.6 g a.i./ha) EPoE at $14 \mathrm{DAS}$, Pendimethalin $30 \mathrm{EC}$ formulation + Imazethapyr 2\% (Ready mix @ 1kg/ha) PE followed by Topramezone 33.6 SC (20.6 g a.i./ha) EPoE at $21 \mathrm{DAS}$.

\section{Acknowledgement}

The authors feel privileged to thank to Dr. G. P. Banjara (Senior Scientist), Department of Agronom, College of Agriculture, IGKV, Raipur (C.G.) for this continuous help, support and guidance throughout this research work.

\section{Reference}

1. Anonymous. A. ICAR Indian Institute of Pulse Research. Kanpur 200249, 2017
2. Anonymous B. Krishi darshika, IGKV, Raipur (C.G.): 2017, 5

3. Ali S, Patel JC, Desai LJ, Singh J. Weed control in Kharif greengram (Vigna radiata L. Wilczek). Bioinfolet. 2013; 10(2A):458-461.

4. Butter GS, Aggarwal N. Efficacy of different herbicides in chickpea under irrigated conditions of Punjab. Indian Journal of weed Sci. 2008; 40: 3(4):169-171

5. Bhutada PO, Bhale VM. Efficacy of herbicides and cultural manegement on weed control in gram (Cicer arietinum). IOSR Journal of Agriculture and Veterinary Science. 2013; 4(5):01-02.

6. Chandel RC, Gupta AK. Two transformation formulas for the generalized multiple hypergeometric function of srivastava and daoust Indian J Pure Appl. Math.1984; 15(6):633-640.

7. Chandrakar S, Sharma A, Thakur DK. Effect of chickpea (Cicer arietinum L.) varieties and weed management practices on quality parameters, nutrient content and 
uptake by crop and weed. International Journal of Agricultural Sciences. 2015; 11(2):217-220.

8. Kour R, Sharma BC, Kumar A, Nandan B, Kour P. Effect of weed management on chickpea (Cicer arietinum) + Indian mustard (Brassica juncea) intercropping system under irrigated conditions of Jammu region. Indian Journal of Agronomy. 2014; 59(2):242-246.

9. Kumar N, Singh KK. Weed management in pulses. Indian Farming. 2010; 60(4):9-12.

10. Mani VS, Malle ML, Gautam KC, Bhagwandas. Weed killing chemicals in potato cultivation. PANS, 1973; 23(8):17-18.

11. Rathod PS, Patil DH, Dodamani BM. Evaluation of time and dose of imazethapyr in controlling weeds of chickpea (Cicer arietinum L.). Legume Research. 2017; 40(5):906-910.

12. Ratnam M, Rao AS, Reddy TY. Integrated Weed Management in Chickpea (Cicer arietinum L.). Indian Journal Weed Science. 2013; 43(1, 2):70-72.

13. Sharma OL. Weed management in chickpea under irrigated conditions of western Rajasthan. Indian Journal of Weed Science. 2009; 41(3, 4):182-184.

14. Singh M, Kumar S, Kumar R, Kumar R. Effects of post emergence herbicides on weed control and yield of field pea and their residual effect on succeeding sorghum and mungbean crop. Legume Research. 2014; 37(4):387394.

15. Singh RP, Verma SK, Singh RK, Idnani LK. Influence of sowing dates and weed management on weed growth and nutrients depletion by weeds and uptake by chickpea (Cicer arietinum) under rainfed condition. Indian Journal of Agricultural Sciences. 2014; 84(4):468-72. 